\title{
Renal histoarchitectural changes in nevirapine therapy: possible role of kolaviron and vitamin $C$ in an experimental animal model.
}

\author{
Ugochukwu Offor ${ }^{1}$, Sunday Adelaja Ajayi ${ }^{1,3}$, Isaac Ayoola Jegede ${ }^{1,2}$, Salem Kharwa ${ }^{1}$, \\ Edwin Coleridge Naidu ${ }^{1}$, Onyemaechi Okpara Azu ${ }^{1,4}$
}

1. Discipline of Clinical Anatomy, School of Laboratory Medicine and Medical Sciences. NelsonR Mandela School of Medicine, University of KwaZulu-Natal, SouthAfrica.

2. Anatomy Department, Faculty of Basic Medical Sciences, College of Health Sciences. Ladoke Akintola University of Technology, Ogbomosho,Nigeria.

3. Department of Anatomy, Faculty of Basic Medical Sciences, College of Medicine. Ekiti State University, Ado-Ekiti, Nigeria.

4. Department of Anatomy, School of Medicine, University of Namibia, Windhoek, Namibia.

\begin{abstract}
Background: There is paucity of literature regarding the nephrotoxicity of antiretroviral drugs and its interaction with plantbased adjuvants. This study investigates the attenuating effect of kolaviron in nevirapine- therapy on the histological structure of the kidneys of adult male Sprague-Dawley rats.

Objective: To determine the attenuating influence of anti-oxidant status of kolaviron on the kidneys of experimental animals following nevirapine administration.

Methods: Forty eight pathogen-free adult male Sprague-Dawley rats were used for this study. The animals were divided into 8 groups (A-H) with 6 animals in each group. Group A was given normal saline as the control; group B was given nevirapine; group $\mathrm{C}$ was given kolaviron; group $\mathrm{D}$ was given vitamin $\mathrm{C}$; group $\mathrm{E}$ was given nevirapine and kolaviron; group $\mathrm{F}$ was given nevirapine and vitamin C; Group $\mathrm{G}$ was given nevirapine and kolaviron (kolaviron withdrawn after day 28) and group $\mathrm{H}$ was given corn oil. The experiment lasted 56 days after which the animals were sacrificed, blood samples were collected through cardiac puncture for serum analysis and the kidneys were harvested and prepared for H\& E histological examination.

Results: Nevirapine caused histoarchitectural damage in the glomerular apparatus with resultant increase in kidney/body weight ratio $(\mathrm{p}<0.001)$. Adjuvant treatment with kolaviron attenuated these nephrotoxic effects. Serum anti-oxidant enzyme (SOD and CAT) activities were significantly reduced in kolaviron and vitamin $\mathrm{C}$ treated animals, whereas in the nevirapine group these parameters were significantly elevated $(\mathrm{P}<0.05)$. However, co-administration of nevirapine and vitamin $\mathrm{C}$ did not improve the histoarchitecture of the kidney.

Conclusion: Adjuvant treatment with kolaviron (an anti-oxidant) for 56 days appears to attenuate the nephrotoxicity of nevirapine in this model.
\end{abstract}

Keywords: Kidney, histoarchitecture, kolaviron, antiretroviral drugs.

DOI: https://dx.doi.org/10.4314/ahs.v17i1.21

Cite as: Offor U, Ajayi SA, Jegede LA, Kharwa S, Naidu EC, Azu OO. Renal histoarchitectural changes in nevirapine therapy: possible role of kolaviron and vitamin C in an experimental animal model. Afri Health Sci. 2017;17(1): 164-174. bttps://dx.doi.org/10.4314/abs.v17i1.21

\section{Introduction}

Nevirapine (Nvp) is the first non-nucleoside reverse transcriptase inhibitor (NNRTI) approved by the U.S. Food
Corresponding author:
Onyemaechi Okpara Azu,
Discipline of Clinical Anatomy,
School of Laboratory Medicine
and Medical Sciences.
Nelson R Mandela School of Medicine,
University of KwaZulu-Natal, South Africa
Email: azu@ukzn.ac.za

and Drug Administration (FDA) for use in combination therapy of Human immunodeficiency virus (HIV-1) infection. Currently, it remains one of the most prescribed antiretroviral drug in developing countries, to prevent vertical transmission (mother-to-child) when used in combination therapy ${ }^{1}$. Despite its therapeutic benefits, treatment with Nvp has been associated with a significant incidence of hepatotoxicity and skin $\mathrm{rash}^{2}$. Sex related differences in the toxicities of Nvp have been reported with incidence of adverse effects being more common in women than in men and this is related to the higher plasma levels in women ${ }^{3,4}$. 
Garcinia kola (GK) seed commonly known as "bitter kola" is the fruit of a tropical plant belonging to the family Guttiferae. It is found in moist forest and grows as a medium sized tree, up to $12 \mathrm{~m}$ high and $1.2 \mathrm{~m}$ wide. It is cultivated and distributed throughout West and Central Africa ${ }^{5}$. GK seed is rich in phytochemicals including Garciniabioflavanonones, xanthones, triterpines and benzophenones with kolaflavonones the major components of kolaviron $^{6}$. GK seeds are traditionally used as remedy for liver disease, heartburn, respiratory inflammation and cough $^{7}$. Anti-diabetic properties have been previously reported ${ }^{8}$ and attributed to the bioflavonoid complex (kolaviron) in GK even in fasted normoglycemic and alloxan-diabetic rats. Iwu' showed that GK biflavanones are pharmacologically active with several pharmacokinetic advantages over simple monomeric flavonoids as they survive firstpass hepatic metabolism which inactivates most flavonoids.

The kidneys play a crucial role in excretion of drugs and may thus are vulnerable to drug toxicities. Drug-induced renal injury contributes up to $25 \%$ of all cases of acute renal failure ${ }^{10}$. Recent interest has focused on complementary and alternative medicines for the treatment of various diseases ${ }^{11}$ and our previous studies ${ }^{8,12}$ support the increasing utilization of plant-based adjuvants for disease management. Many of the biological functions of flavonoids and phenolic compounds have been attributed to their free radical scavenging and anti-oxidant activities $^{12,13}$. Studies have also shown the ability of kolaviron $(\mathrm{kv})$ to protect against various noxious compounds like phalloidin, amanita, 2-acetylaminofluorene, carbon tetrachloride, paracetamol, aflatoxin and dimethyl nitrosamine in the liver of rodents ${ }^{14}$. With paucity of data reporting potential attenuating influence of kolaviron in any antiretroviral treatment and in view of the potential side effects of Nvp, the understanding of the mechanism(s) for this side effect should allow for further investigation of the adjuvant use of kolaviron to ameliorate the effects. Based on these considerations, this work investigates the histo-architectural effect of kolaviron on the kidneys of Nvp-treated male Sprague-Dawley rats comparing this with known anti-oxidant vitamin $\mathrm{C}$ in an experimental animal model.

\section{Materials and method Chemicals}

Nvp (Aspen) and vitamin C were obtained from Pharmacare Ltd, Port Elizabeth, South Africa and were of analytical grade.

\section{Plant materials}

GK seeds were purchased from Eda-Oniyo Ekiti market, Ekiti State, Nigeria. The seeds were taken to the Herbarium unit of the Department of Botany, Obafemi Awolowo University, Ile-Ife, Nigeria for authentication. Samples of it are retained at the herbarium for future reference and allocated voucher specimen number NHI 16745.

\section{Isolation of Kolaviron}

$\mathrm{Kv}$ was isolated according to the published procedure of ${ }^{9}$ and modified by ${ }^{15}$. The outer cover of GK seeds were peeled, sliced and air-dried in the laboratory $\left(25-28^{\circ} \mathrm{C}\right)$ and ground into powdered form. The powdered seeds were extracted with n-hexane, in a soxhlet extractor for 24hr. The defatted, dried marc was repacked and then extracted with methanol in a soxhlet extractor. The extract was concentrated and diluted to twice its volume in distilled water and extracted with ethylacetate $(6 \times 300 \mathrm{ml})$. The concentrated ethylacetate yielded kolaviron, a golden yellow solid.

\section{Ethical approval}

The study protocol was approved by the University of KwaZulu Natal Animal Ethics Committee (Ethical clearance number: Animal/102/14). The animals were bred at the Biomedical Resource Unit (BRU) animal facility of the University of KwaZulu Natal Westville campus, Durban-South Africa. All animals received humane care in accordance with the criteria outlined in the 'Guide for the Care and Use of Laboratory Animals' prepared by the National Academy of Science (NAS) and published by the National Institute of Health ${ }^{16}$.

\section{Animal model}

Forty eight pathogen-free adult male Sprague-Dawley rats aged 9-10 weeks old and weighing approximately 200-250g were used for this study. They were kept in well ventilated plastic cages $(36 \mathrm{~cm}$ long $\times 24 \mathrm{~cm}$ wide and $15 \mathrm{~cm}$ high) under standard animal house conditions at room temperature $\left(28-30^{\circ} \mathrm{C}\right)$ and were fed with standard pelleted rat chow from (Meadow feeds a Division of Astral Operations Limited, Durban, South Africa) and given tap water ad libitum.

\section{Experimental design}

The animals were randomly distributed to eight treatment groups: A, B, C, D, E, F, G, H with six animals per group $(n=6)$. The animals were allowed 2 weeks of acclimatization before the treatment commenced. Group A received

African Health Sciences Vol 17 Issue 1, March, 2017 
normal saline as the control, group B were given Nvp at a dose of $1.54 \mathrm{mg} / \mathrm{kgbw}$, Group $\mathrm{C}$ were given $\mathrm{Kv}$ at a dose of $200 \mathrm{mg} / \mathrm{kgbw}$, Group D were given vitamin C at a dose of $250 \mathrm{mg} / \mathrm{kgbw}$, Group E were given Nvp at a dose of $1.54 \mathrm{mg} / \mathrm{kgbw}$ and $\mathrm{Kv}$ at a dose of $200 \mathrm{mg} / \mathrm{kgbw}$, Group F were given Nvp at a dose of $1.54 \mathrm{mg} / \mathrm{kgbw}$ and vitamin $\mathrm{C}$ at a dose of $250 \mathrm{mg} / \mathrm{kgbw}$, Group $\mathrm{G}$ were given Nvp at a dose of $1.54 \mathrm{mg} / \mathrm{kgbw}$ and $\mathrm{Kv}$ at a dose of $200 \mathrm{mg} /$ kgbw (kolaviron was withdrawn after day 28). Group H were given corn oil which served as the vehicle for the administration of $\mathrm{Kv}$. All administration was done daily by oro-gastric gavage. The experiment lasted for 56 days.

\section{Histological examination of tissues}

Twenty four hours after the last treatment the animals were humanely sacrificed under excess flouthane ${ }^{\circledR}$ anaesthesia and the kidneys removed and weighed. They were examined for gross pathology and immediately fixed in $10 \%$ formal saline. After fixation, the tissues were dehydrated in a graded series of alcohol, cleared in Xylene and embedded in paraffin wax using a cassette. The tissues were sectioned at $4 \mu \mathrm{m}$ thickness using LeicaR RM 2255 microtome and stained with hematoxylin and eosin. The stained slides were cover slipped using DPX mounting glue directly over the tissue section ensuring no air bubbles were trapped. Thereafter, the slides were left overnight to dry and scanned using a Leica SCN 400 (Leica Microsystems GmbH, Wetzlar, Germany) and measured at 250 magnification using image analyser Leica (DMLB) and Leica QWIN software.

\section{Biochemical assays}

Blood samples were collected through cardiac puncture and allowed to clot for 30 minutes and then centrifuged for 15 minutes at $3000 \mathrm{rpm}$. The serum was decanted into Eppendoff tubes and preserved at $-84^{\circ} \mathrm{C}$ prior to biochemical analysis. SOD levels in the serum were assayed according to the method of Das et $\mathrm{al}^{17}$. CAT activity was assayed using hydrogen peroxide as substrate according to the method of Aebi ${ }^{18}$.

\section{Statistical analysis}

The data obtained from all the groups was compiled and statistically analyzed and expressed as mean \pm SEM. Since we had a parametric data, differences between groups were compared using One-way ANOVA, with $\mathrm{p}<0.05$ considered significant.

\section{Results}

Mortality: No animal died during the experimental period.

Effects of Nevirapine and Kolaviron on kidney/ body weight changes:

There was a significant increase in kidney-body weight ratio $\mathrm{p}<0.001$ in nevirapine-treated animals whereas animals in group $\mathrm{C}$ treated with kolaviron for same duration did not show any deleterious effect. Further, group $\mathrm{D}$ treated animals given vitamin $\mathrm{C}$ showed no increase in kidney-body weight ratio significant at $\mathrm{p}<0.05$. But when treated concomitantly with nevirapine in group F, we observed significant increase in kidney-body weight ratio $\mathrm{p}<0.001$. Treatment of nevirapine and kolaviron in group $E$ for 56 days showed significant improvement $p<0.05$. In contrast, treatment of nevirapine and kolaviron in group $G$ where kolaviron was withdrawn after day 28 showed a significant increase in kidney -body weight ratio $\mathrm{p}<0.001$. Group $\mathrm{H}$ administered with corn oil showed no renal injury significant at $\mathrm{p}<0.05$ (See table 1). 
Table 1: Kidney and body weight changes across the groups

\begin{tabular}{|l|l|l|l|l|l|} 
Groups & Initial BW(g) & Final BW(g) & BW Diff (\%) & \multicolumn{1}{l}{ KW(g) } & KWBR ( $\left.\times 10^{-3}\right)$ \\
\hline A & $213.5 \pm 0.11$ & $380.0 \pm 5.34$ & $166.5(77.99)$ & 2.11 & $5.55 \pm 0.08$ \\
B & $220.5 \pm 5.73$ & $417.0 \pm 5.5$ & $196.5(89.12)$ & $2.40^{*}$ & $5.73 \pm 0.18^{*}$ \\
C & $202.5 \pm 0.31$ & $356.5 \pm 4.49$ & $154.0(76.05)$ & 1.99 & $5.59 \pm 0.12$ \\
D & $243.0 \pm 0.42$ & $392.0 \pm 4.20$ & $149.0(61.32)$ & $1.97^{*}$ & $5.03 \pm 0.13$ \\
E & $198.5 \pm 0.03$ & $402.5 \pm 5.23$ & $204.0(102.77)$ & 2.13 & $5.29 \pm 0.06$ \\
F & $246.5 \pm 0.09$ & $397.5 \pm 5.70$ & $151.0(61.26)$ & $2.34 *$ & $5.88 \pm 0.08 * *$ \\
G & $232.0 \pm 0.17$ & $390.0 \pm 5.29$ & $158.0(68.10)$ & 2.20 & $5.62 \pm 0.09$ \\
H & $212.5 \pm 0.13$ & $390.0 \pm 5.12$ & $177.5(83.53)$ & 2.10 & $5.37 \pm 0.08$ \\
\hline
\end{tabular}

Key:

$\mathrm{BW}=$ Body weight, $\mathrm{KW}=$ Kidney weight, $\mathrm{KWBR}=$ Kidney weight body ratio; $\left.{ }^{*} \mathrm{P}<0.05 ; * * \mathrm{P}<0.01\right)$

Effects of Nevirapine and Kolaviron on antioxidant enzymes

Catalase, which acts as a preventive anti-oxidant enzyme plays an important role in protection against the delete- rious effects of lipid peroxidation was significantly decreased following treatment with Nvp alone $(\mathrm{p}<0.01)$ and in group with withdrawal of $\mathrm{Kv}(\mathrm{p}<0.05)$ respectively (Fig 1).

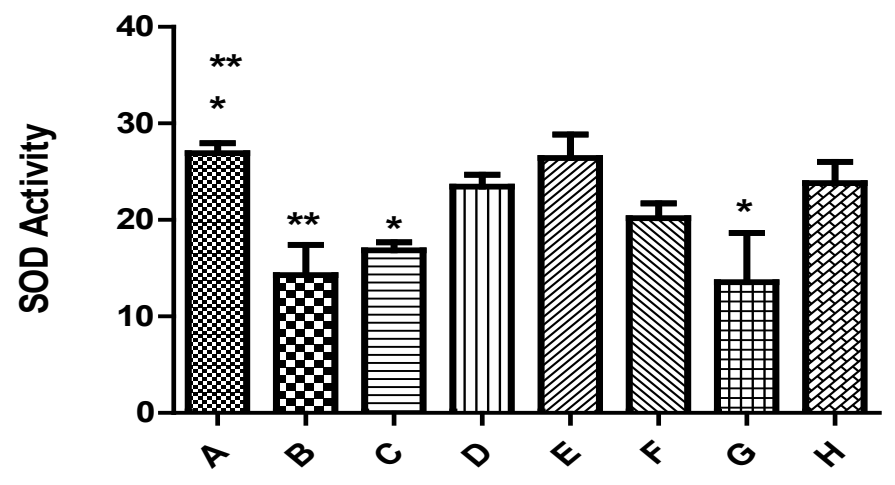

Fig 1: Graphical representation of SOD activity.

Though the activities of SOD in Kv and vitamin C treated groups were not significantly higher than positive con- trol, Nvp treated group was much lower in value than the control (Fig. 2). 


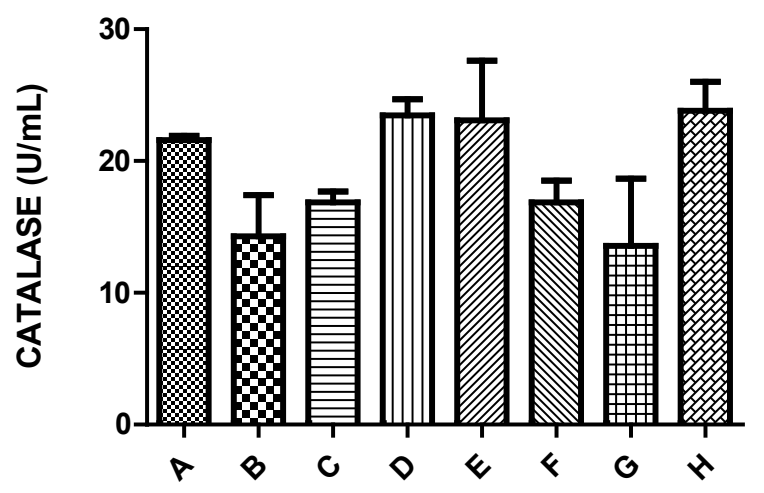

Fig 2: Graphical representation of CAT activity.

Effects of Nevirapine and Kolaviron on the histological changes of thekidney

Histopathological sections of kidneys in control rats revealed adequate preservation of tubular structures with the presence of glomerular capsule. There were distortions in the kidney histology of nevirapine treated animals with necrosis of glomeruli and atrophy of the proximal and distal convoluted tubules (plate B).

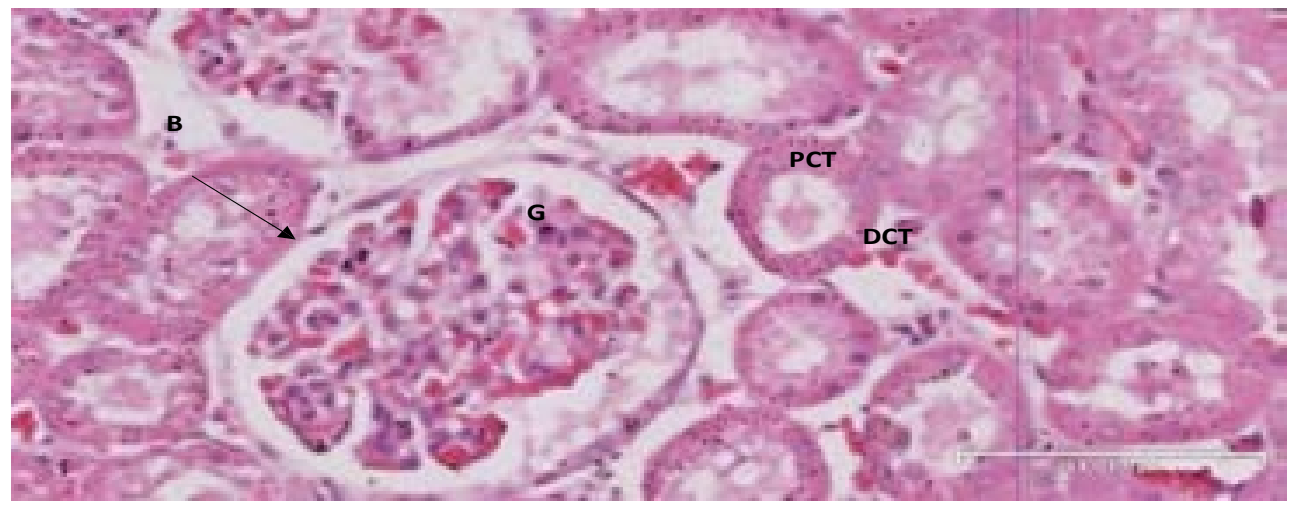

Plate 1: Cross section of kidney of Sprague-Dawley rats in control group. Normal histoarchitecture displayed. (H \& Estains).

$\mathrm{B}=$ Bowmans capsule, $\mathrm{PCT}=$ Proximalconvolutedtubules, $\mathrm{DCT}=$ Distal convulted tubules, $\mathrm{G}=\mathrm{Glomerus}$.

B

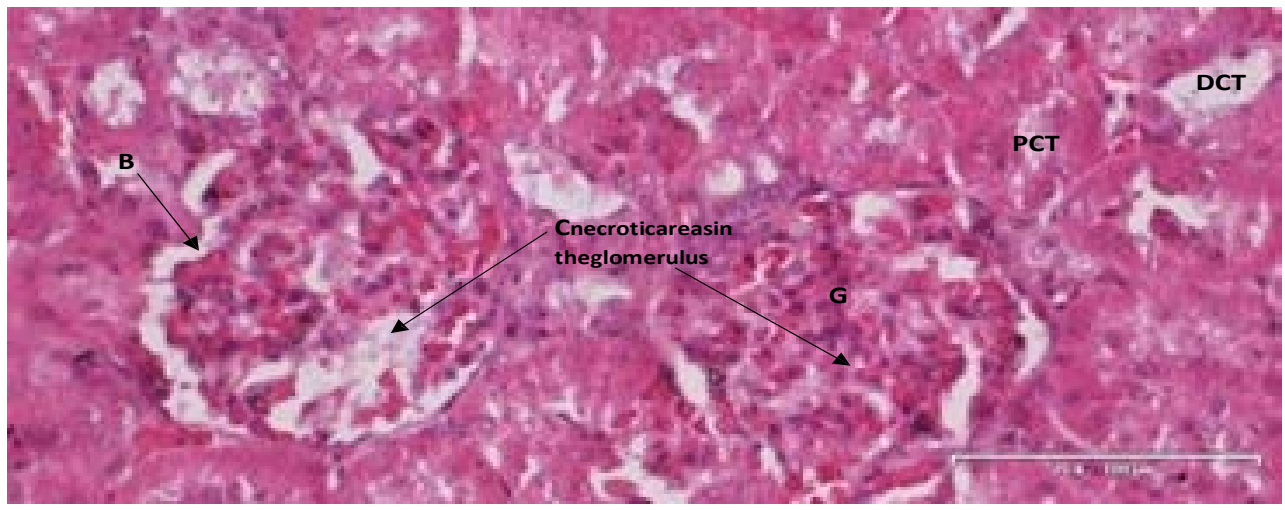

Plate 2: Cross section of kidney of Sprague-Dawley rats treated with nevirapine only. Note the necrosisinglomeruli, atrophied PCT and DCT. (H \& Estains).

G=Glomerulus, DCT=Distal convoluted tubule PCT=Proximal convoluted tubules, B=Bowman's space, $\mathrm{C}=$ Capillary loops.

C 
These changes were markedly generalized. There were no observable changes in the histology of the kidney of the groups treated with kolaviron alone, vitamin $\mathrm{C}$ alone and $\mathrm{Kv}$ with $\mathrm{Nvp}$ as these compared favourably with controls (plates 3, 4, 5). Adjuvant treatment with $\mathrm{Kv}$ and $\mathrm{Nvp}$ in group $\mathrm{E}$ showed an improved histological layout of the kidney (plate 5) whereas group G treated with Nvp and
$\mathrm{Kv}(\mathrm{Kv}$ withdrawn after day 28) showed cellular distortion of the glomeruli with atrophy of Bowman's capsule and space and a generalized hypocellularity (plate 7). Histoarchitectural kidney sections following vitamin $\mathrm{C}$ and Nvp co-treatment showed disorganization of the proximal and distal convoluted tubules with generalized hypoplasia (plate 6).

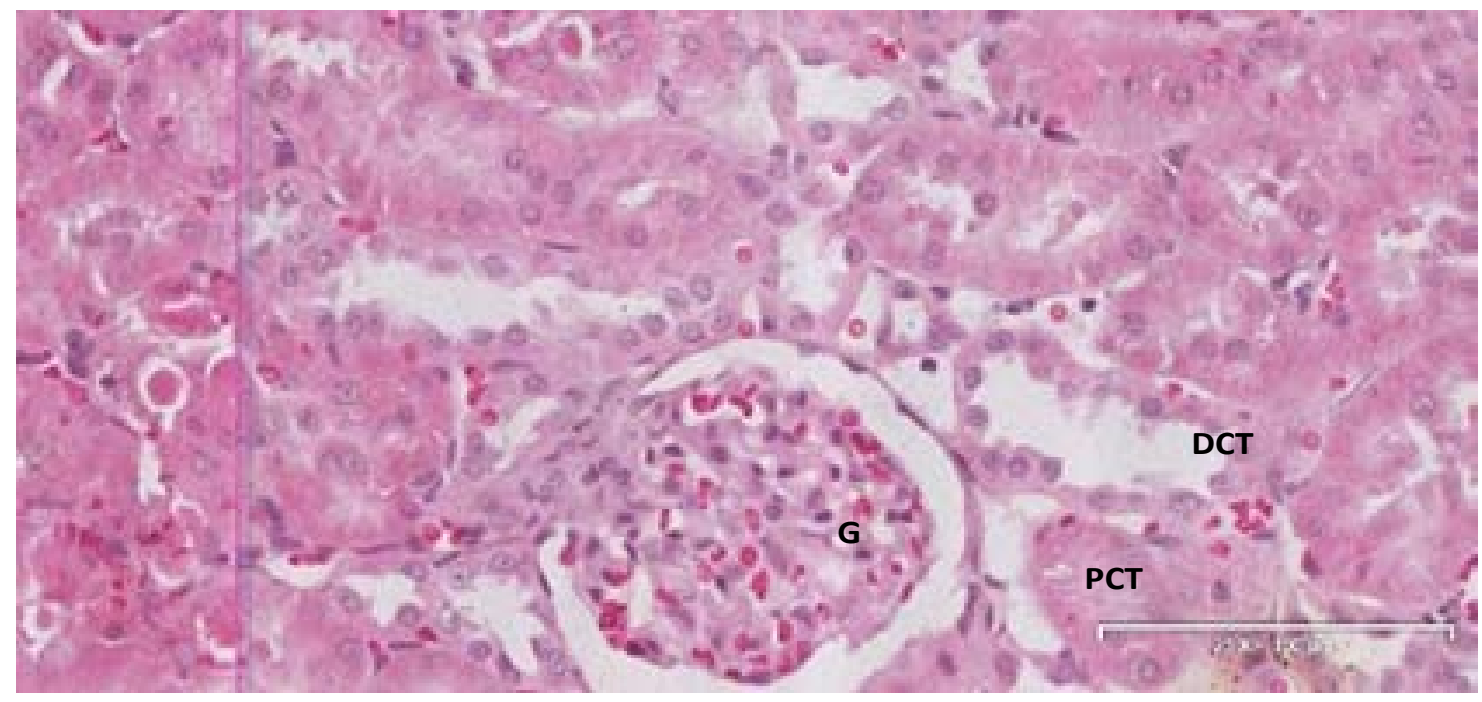

Plate 3: Cross section of kidney of Sprague-Dawley treated with kolaviron only. Note:

Histoarchitecture normal. (H \& Estains).

G=Glomerulus, PCT=Proximalconvolutedtubules, $\mathrm{DCT}=$ Distalconvolutedtubules, $\mathrm{C}=$ Capillary loops.

D

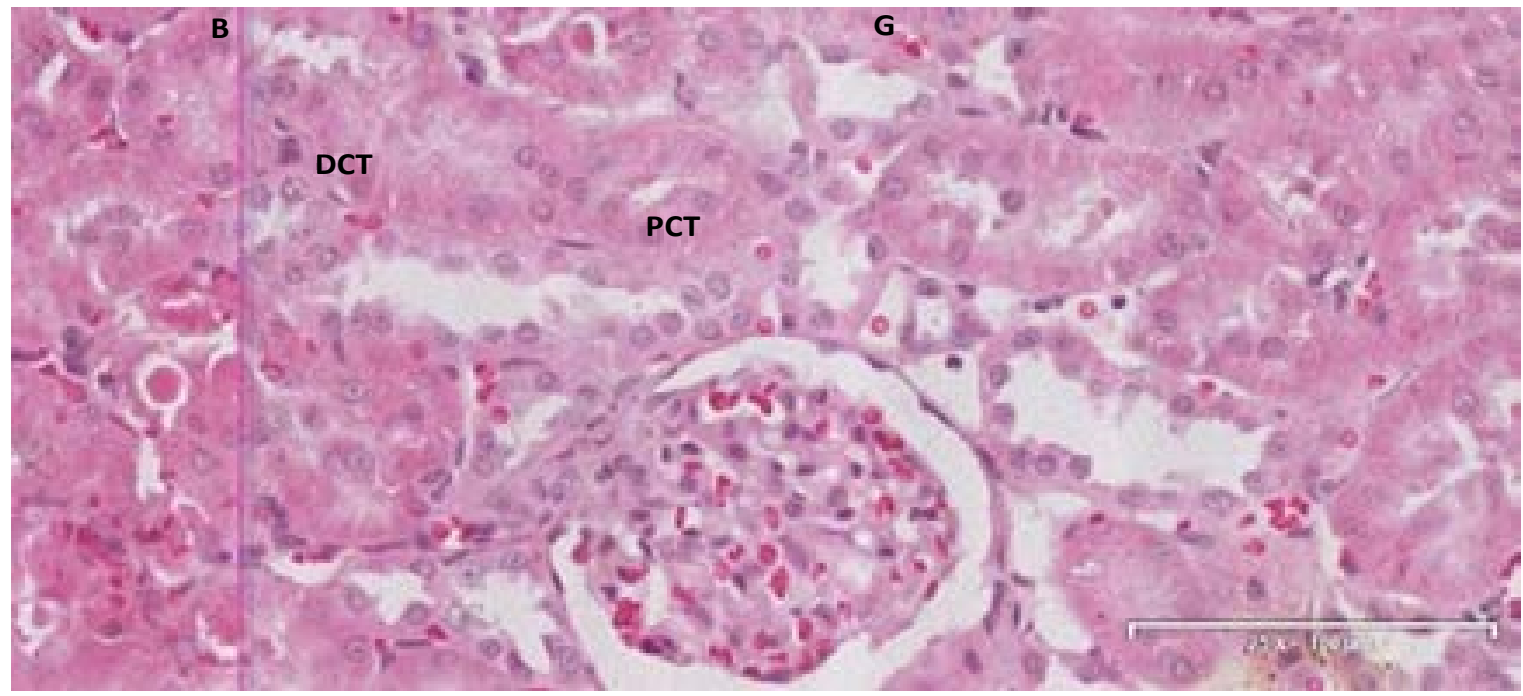

Plate 4: Cross section of kidney of Sprague-Dawley rats treated with vitamin C only.

Histoarchitecture normal. (H \& Estains).

B=Bowman'sspace,G=Glomerulus,DCT=Distalconvolutedtubules, $\mathrm{PCT}=$ Proximal convoluted tubules. 


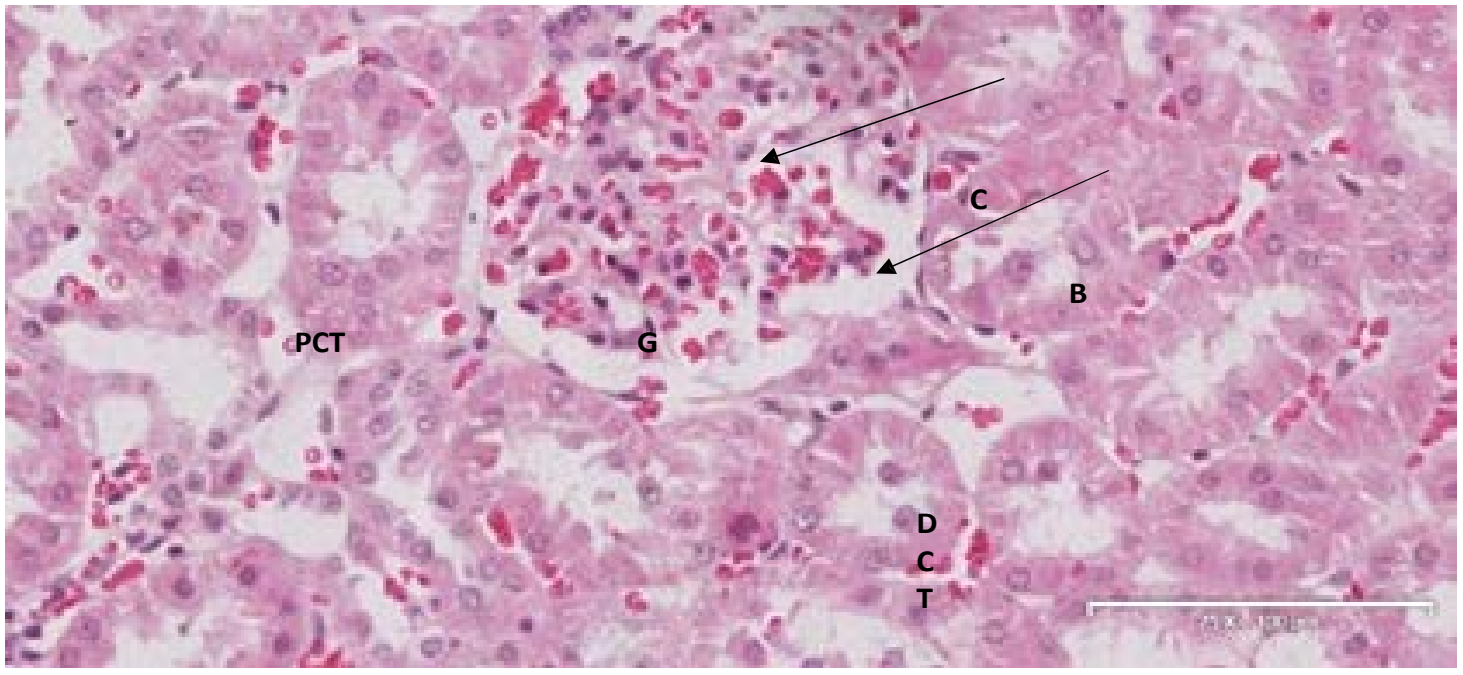

Plate 5: Cross section of kidney of Sprague-Dawley rats treated with nevirapine and kolaviron. Histoarchitecture normal. (H \& Estains).

G=Glomerulus, C=Capillary loops, B=Bowman'sspace, $\mathrm{DCT}=$ Distal convoluted tubules, $\mathrm{PCT}=\mathrm{Proximal}$ convoluted tubules.

$\mathbf{F}$

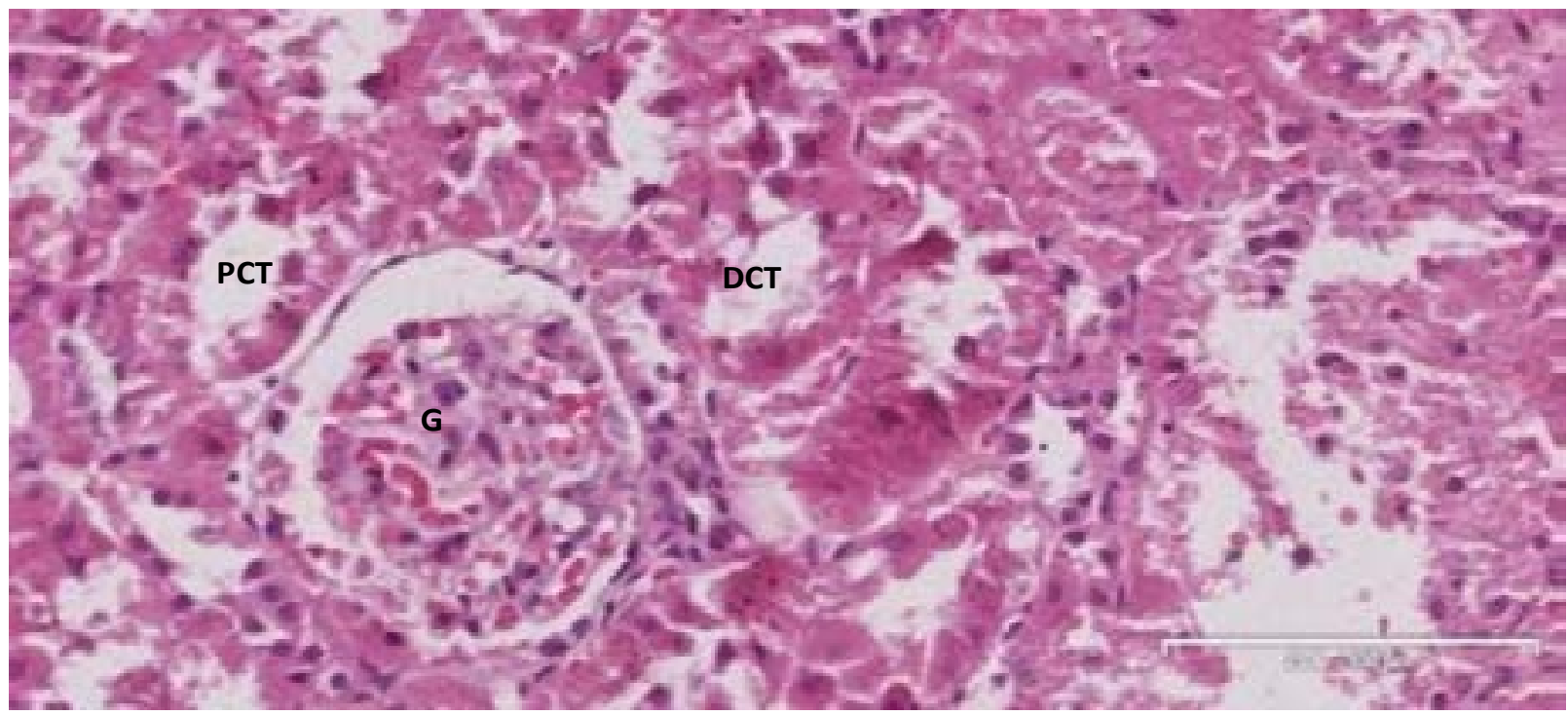

Plate 6: Cross section of kidney of Sprague-Dawley rats treated with nevirapine and vitamin C. Note: Disorganization in PCT \& DCT cellular architecture with generalized hypoplasia. (H \& Estains). PCT=Proximal convoluted tubules, DCT=Distal convoluted tubules. 


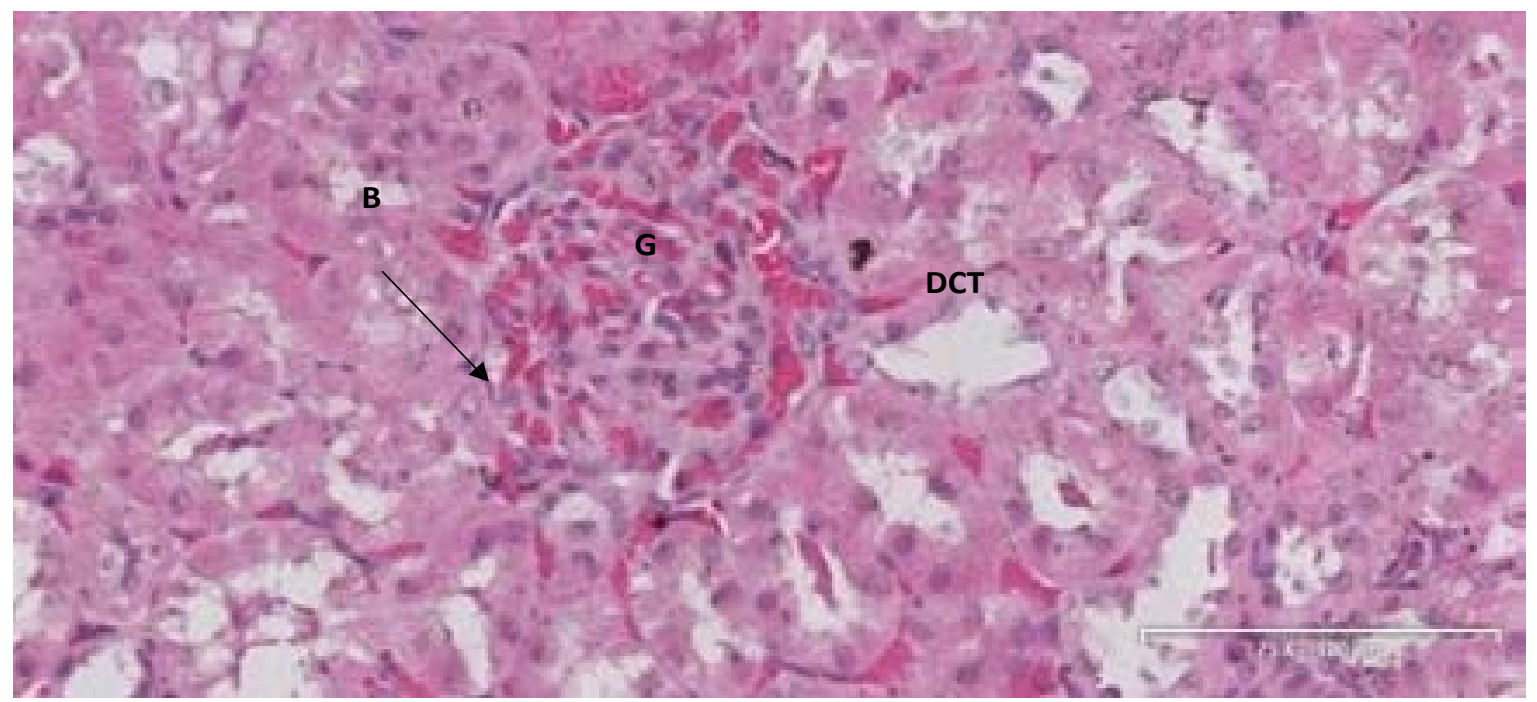

Plate7: Cross section of kidney of Sprague-Dawley rats treated with nevirapine and kolaviron (where kolaviron was withdrawn after 28 days).Note: Cellular distortion of glomeruliwithatrophy of Bowman's capsule and space. Generalized hypocellularity. (H \& Estains). G=Glomerulus, B=Bowman's capsule, DCT=Distal convoluted.

H

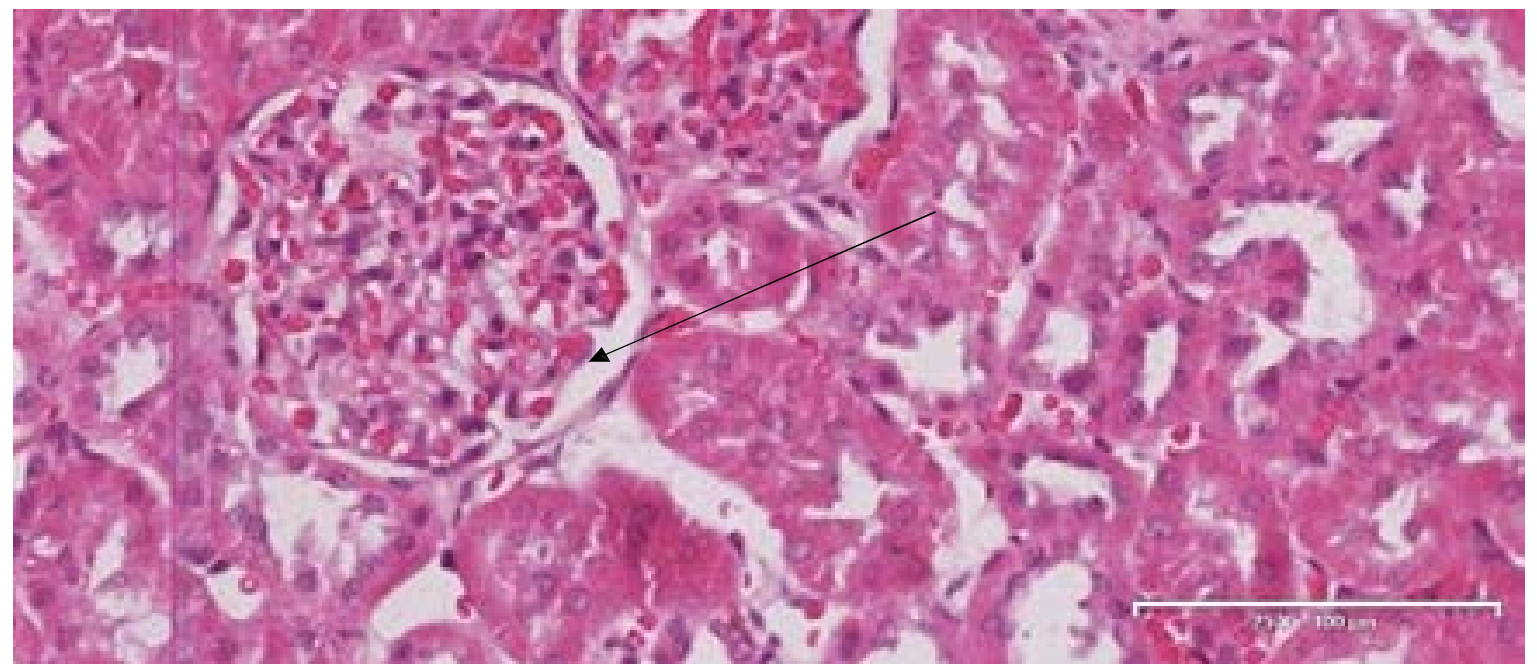

\section{Plate8: Cross section of kidney of Sprague-Dawley rats given cornoil. Histoarchitecture essentially normal.(H \& Estains). \\ $\mathbf{G}=$ Glomerulus,B=Bowman'sspace, $\mathbf{D C T}=$ Distalconvolutedtubules,PCT=Proximalconv oluted tubules.}

\section{Discussion}

The kidney plays a major role in the maintenance of constant volume and composition of the extracellular fluid hence does the basic functions of glomerular filtration, tubular reabsorption and tubular secretion ${ }^{19}$. Kidney function can be evaluated by a number of methods. Male rats are particularly a suitable animal model for evaluating tubular lesions because their intra-renal enzyme distribution is similar to that in $\operatorname{man}^{20}$.
Antiretroviral therapy (ART) using Nvp has led to improved wellness of HIV-infected patients by lowering the viral load and hence their positive impact on longevity. However this has come with its potential toxicities on body organs such as the liver with altered oxidative stress status implicated ${ }^{21}$. Histological studies are important in anatomical localization of the toxic or the beneficial actions of various drugs. In this study, the histoarchitecture of the glomeruli, proximal and distal convolut-

African Health Sciences Vol 17 Issue 1, March, 2017 
ed tubules, present a compromised histological state in the Nvp-treated group with extensive necrosis as well as atrophy of Bowman's capsule and space. Morphological and physiological studies have identified the renal tubule systems as the site of maximum damage by toxicants and hence any direct protective effect would be apparent on the tubule system. This has been observed in the groups treated with $\mathrm{Kv}$ as well as vitamin $\mathrm{C}$ whereupon this parameter was restored. Our current work on the testes also confirms the deleterious histological changes following ART on testicular tissue ${ }^{22}$.

Oxidative stress has been recognized as a major pathway of action for most antiretroviral drugs via various mechanisms that may trigger it ${ }^{23}$. Anti-oxidant enzymes are inhibited by toxic drugs including Nvp and renal activities of catalase and superoxide dismutase are reduced in this experiment. The reactive oxygen species (ROS) produced directly act on cell components including lipids, proteins and DNA and destroy their structure. The consequence of this is oxidative stress and cell death. $\mathrm{Kv}$ is rich in biflavonoids which has been ascribed strong anti-oxidants properties $^{24}$. It could possibly have a reno-protective effect in Nvp-treated rats by exerting its beneficial effects via modulating the anti-oxidant system. Together, the results suggest the beneficial effects of using a free radical scavenger in modulating Nvp-associated nephrotoxicty. The use of vitamins ( $\mathrm{C}$ and $\mathrm{E}$ ) as anti-oxidant supplements in ART has been practiced both in experimental and clinical conditions because they act as 'mop-up' for generated free radicals in the milieu. The effects of $\mathrm{Kv}$ and vitamin $\mathrm{C}$ in this protocol have tilted towards similarities adjudged from the various parameters assessed. Oxidative stress-induced lipid peroxidation generates numerous electrophilic aldehydes that can attack many cellular targets. They may also inhibit drug-induced apoptosis by inactivating caspase activity which would diminish the efficacy of treatment. The use of anti-oxidants during therapy may enhance therapy by reducing the generation of oxidative-stress induced aldehydes ${ }^{25}$.

Weight coefficient (organ/BW ratio) is a sensitive, effective and economic indicator of the toxicology and thus important in the identification of target organ by toxicants $^{26}$. Our investigation reveals that the weight coefficient of kidney tissue in Nvp-treated animals was signifi- cantly higher than those of controls, further supporting the toxicity of Nvp to this organ. Renotoxicity just like testicular toxicity can manifest in the form of organ swelling, atrophy or hypertrophy ${ }^{27}$ and the significantly increased KW in Nvp-treated group is consistent with this assertion possibly because of high cytotoxicity-induced tubulo-glomerular atrophy.

While these reports buttress our hypothesis that when massive cellular loss occurs, there is a sharp decline in morphometric parameters which are largely attenuated by anti-oxidant treatment ${ }^{23}$, we believe that the precise putative explanations for the structural changes observed may be better explained using stereological techniques in order to generate a 3-dimensional approach. That way a more detailed quantification can be done with the aim of generating relevant approaches to the mechanisms underlying the processes. More so, therapeutic approaches with anti-oxidants in HIV/AIDS treatment presents a challenge to researchers because AIDS itself is a vexing disease with many implications. Therefore, the understanding of the mechanistic information regarding the interactions of the various modalities of biological and exogenous anti-oxidants in the course of therapy would significantly broaden the scope of treatment with consequent reduction of the morbidity and mortality associated with HIV/AIDS.

\section{Conclusion}

While studies to elucidate histo-architectural changes in the osmo-regulatory organ (kidney) following antiretroviral therapy is limited, we have highlighted in our experiment that treatment with Nvp disrupts renal histo -architecture leading to compromise in the integrity and biochemical functioning. These perturbations are largely attenuated by adjuvant treatment with $\mathrm{Kv}$ which compares favourably with known anti-oxidant (vitamin C) in this experimental animal model. However, further studies (using stereological techniques) are needed to explain the observed effects and provide a mechanistic explanation for $\mathrm{Kv}$ action.

\section{Aknowledgement}

We thank the School of Laboratory Medicine and Medical Sciences and the College of Health Sciences, University of KwaZulu-Natal Durban South Africa for the financial support to the first author and the Post-Doctoral grant awarded to the second author. 


\section{Conflict of interest}

All authors declare that there are no conflicts of interest whatsoever.

\section{References}

1. Lockman S, Shapiro RL, Smeaton LM, et al. Response to antiretroviral therapy after single, peripartum dose of nevirapine. N.Engl. J. Med. 2007; 356:135-147 PubMed .

2. Pollard RB, Robinson P, Dransfield K. Safety profile of nevirapine, a nonnucleoside reverse transcriptase inhibitor for the treatment of human immunodeficiency virus infection. Clin Ther. 1998; 20:1071-1092 PubMed .

3. Bersoff-Matcha SJ, Millier WC, Aberg JA, van Der Horst C, Hamrick HJ, Powderly WG. Sex differences in nevirapine rash. Clin Infect Dis. 2001; 32(1):124-139. PubMed

4. Ofotokun I, Chuck SK, Hitti JE. Antiretroviral pharmacokinetic profile: a review of sex differences. Gend Med. 2007; 4(2): 106-119. PubMed

5. Kabungu L, Gale FC, Aonzo E, Nicoletti M, Messana I. New biflavonone from the bark of G.kola. Planta Medica. $1987 ; 275$.

6. Iwu MM, Igboko OA, Okunji CO, Tempesta MS. Antidiabetic and aldose reductase activities of biflavanones of Garcinia kola. J. Pharm. Pharmacol. 1990; 42:290-292 PubMed .

7. Orie NN, Ekon EU. The bronchodialatory effect of Garcinia kola. East Africa Med J. 1993; 73:143-145 PubMed .

8. Azu OO, Osinubi AA, Noronha CC, Okanlawon AO. Hypoglycaemic activities of extract of Garcinia kola seeds in normal, hyperglycaemic and alloxan-induced diabetic rats. West Afri J. anat. 2005; 8: 141-149 PubMed .

9. Iwu MM. Antihepatotoxic constituents of Garcinia kola seeds. Experiential. 1985; 41:699-700 PubMed .

10. Bentley ML, Corwin HL, Dasta J. Drug-induced acute kidney injury in the critically ill adult: recognition and prevention strategies. Crit Care Med. 2010; 38(6):169 PubMed -174.

11. Aruoma OI, Sun B, Fuji H, et al. Low molecular proanthocyamidin dietary biofactor oligonol: Its modulation of oxidative stress, bioefficacy, neuroprotection, food application and chemoprevention potentials. Biofactors. 2006; 27:245-265 PubMed .

12. Azu OO, Duru FIO, Osinubi AA, Noronha CC, Elesha SO, Okanlawon AO. Preliminary studies on the antioxidant effect of Kigelia africana fruit extract (Bignoniaceae) in male Sprague-Dawley rats. African Journal of Biotechnology. 2010; 9(9): 1374-1381.

13. Seef LB, Lindsay KL, Bacon BR, Kresina TF, Hoofnagle $\mathrm{JH}$. Complementary and alternative medicine in chronic liver disease. Hepatology. 2001; 34:595-603 PubMed .

14. Farombi EO, Tahnteng JG, Agboola O, Nwankwo JO, Emerole GO. Chemoprevention of 2-acetyl aminofluorene-induced hepatotoxicity and lipid peroxidation in rats bykolaviron. A Garcinia kola seed extract. Food Chem.Toxicol. 2000; 38:535-541 PubMed .

15. Braide VB. Antihepatotoxic biochemical effects of kolaviron, a biflavonoid of Garcinia kola seeds. Phytother. Res. 1991; 5:35-37 PubMed.

16. National institutes of Health Guide for the care and use of Laboratory Animals. DHEW Publication (NIH) (1985): Revised, Office of Science and Health Reports, DRR/NIH, Bethesda; USA.

17. Das K, Samanta L, Chainy G.B.N (2000). A modified spectrophometric assay of superoxide dismutase using nitrite formation of superoxide radicals. Indian J. Biochem. Biophys. 37: 201-204.

18. Aebi H (1974). Catalase. In: Methods of enzymatic analysis. Vol. II, Bergmayer, H.U. (Ed.), Academic Press, New York, pp. 673-683.

19. \&nbs; Skalova S. The diagnostic role of urinary $\mathrm{N}$-acetyl-b-glucosaminidase (NAG) activity in the detection of renal tubular impairment. Acta Medica 2005; 48:75-;80. PubMed

20. Raab RP. Diagnostic value of urinary enzyme determinations. Clin Chim Acta 1972; 18:15-22. PubMed

21. Adaramoye OA, Adesanoye OA, Adewumi OM, Akanni O. Studies on the toxicological effect of nevirapine, an antiretroviral drug on liver, kidney and testis of male wistar rats. Human Exp Toxicol. 2012; 31:676.

22. Azu OO, Naidu ECS, Naidu JS, et al. Testicular histomorphologic and stereological alterations following short-term treatment with highly active antiretroviral drugs (HAART) in an experimental animal model. Journal Andrology. 2014; 2(5): 772-779. PubMed

23. Azu OO, Duru FIO, Osinubi AA, Oremosu AA, Norohna CC, Okanlawon AO \& Elesha SO. (2010) Histomorphometric effects of Kigelia Africana (Bignoniaceae) fruit extract on the testis following short-term treatment with cisplatin in Sprague-Dawley rats. Middle East FertilSoc J 15, 200-208. 
24. Farombi EO, Nwaokeafor IA. Anti-oxidant mechanism of action of kolaviron: Studies on serum lipoprotein oxidation, metal chelation and oxidative microsomal membrane damage in rats. Clin. Exp. Pharmacol. Phys. 2005; 32:667-674 PubMed .

25. Conklin KA. Chemotherapy-associated oxidative stress: impact on chemotherapeutic effectiveness. Integrative Cancer Therapies 2004; 3:294-300.
26. Asagba SO, Adaikpoh MA, Kadiri H \& Obi FO. (2007) Influence of aqueous extract of Hibiscus sabdariffa L. petal on cadmium toxicity in rats. Biol Trace Elem Res 115, 47-57.

27. Afolayan AJ \& Yakubu MT. (2009) Effect of Bulbine natalensis Baker stem extract on the functional indices and histology of the liver and kidney of male Wistar rats. J Med Food 12, 814-820. 